\title{
EXTENSION AND LIFTING OF OPERATORS AND POLYNOMIALS
}

\author{
JESÚS M.F. CASTILLO, RICARDO GARCÍA, AND JESÚS SUÁREZ
}

\begin{abstract}
We study the problem of extension and lifting of operators belonging to certain operator ideals, as well as that of their associated polynomials and holomorphic functions. Our results provide a characterization of $\mathcal{L}_{1}$ and $\mathcal{L}_{\infty}$-spaces that includes and extends those of Lindenstrauss-Rosenthal [32] using compact operators and González-Gutiérrez [23] using compact polynomials. We display several examples to show the difference between extending and lifting compact (resp. weakly compact, unconditionally convergent, separable and Rosenthal) operators to operators of the same type. Finally, we show the previous results in a homological perspective, which helps the interested reader to understand the motivations and nature of the results presented.
\end{abstract}

\section{INTRODUCTION}

Many authors have considered the problem of extension and lifting of operators 19, 20, 28, 29, 31, 32, 33, 39, of homogeneous polynomials 22, 11, 15, 18, 23, and holomorphic mappings [1, 2, 11, 18, 28, 40] in Banach spaces. We present here a unifying method of proof for most of those results, and several new ones, using some tools of homological algebra.

To this end, recall that a short exact sequence of Banach spaces and linear continuous operators is a diagram

$$
0 \longrightarrow Y \stackrel{i}{\longrightarrow} X \stackrel{q}{\longrightarrow} Z \longrightarrow 0
$$

where the image of each arrow coincides with the kernel of the following one. The open mapping theorem ensures that $Y$ must then be a subspace of $X(i$ is an injection) and $Z$ is the corresponding quotient $X / Y$ ( $q$ is a quotient map). The exact sequence is said to split if $Y$ is complemented in $X$; which means that there is a linear continuous projection $p: X \rightarrow Y$. Let $\mathfrak{L}$ denote the class of all linear continuous operators. The projection $p$ is, by definition, a linear continuous extension of the identity of $Y$ and thus " $Y$ is complemented in $X$ " is equivalent to "for every Banach space $E$ every linear continuous operator $t \in \mathfrak{L}(Y, E)$ can be extended to a linear continuous operator $T \in \mathfrak{L}(X, E)$ through $i$; i.e., $T i=t$ ". It is part of the folklore -see [13, 1.1]- that this is equivalent to "for every Banach space $V$ every linear continuous operator $t \in \mathfrak{L}(V, Z)$ can be lifted to a linear continuous operator $T \in \mathfrak{L}(V, X)$ through $q$; i.e., $q T=t^{\prime \prime}$.

This research has been supported in part by Project MTM2010-20190-C02-01 and Junta de Extremadura GR10113 "IV Plan Regional I+D+i, Ayudas a Grupos de Investigación" .

2010 Mathematics Subject Classification: 46B28, 46G20, 46M10,46A16.

Key words and phrases: Banach spaces, operator ideals, extension/lifting of operators, polynomials and holomorphic mappings, short exact sequences of Banach spaces, exact functors. 
We are interested in considering the situation when we replace the class $\mathfrak{L}$ by another class $\mathcal{A}$ of operators, polynomials or holomorphic mappings acting between Banach spaces.

Definition 1. We will say that an exact sequence $0 \rightarrow Y \stackrel{i}{\rightarrow} X \stackrel{q}{\rightarrow} Z \rightarrow 0 \mathcal{A}$-splits if, for every Banach space $E$, every $t \in \mathcal{A}(Y, E)$ can be extended to a $T \in \mathcal{A}(Y, E)$ through $i$; i.e., $T i=t$. We will say that it $\mathcal{A}$-lifts if, for every Banach space $V$, every $t \in \mathcal{A}(V, Z)$ can be lifted to a $T \in \mathcal{A}(V, X)$ through q; i.e., $q T=t$.

We will say that an exact sequence $0 \rightarrow Y \stackrel{i}{\rightarrow} X \stackrel{q}{\rightarrow} Z \rightarrow 0$ uniformly $\mathcal{A}$-splits when there exists $\lambda>0$ such that, for every Banach space $V$, every $t \in \mathcal{A}(Y, V)$ can be extended to a $T \in \mathcal{A}(Y, V)$ through $i$ with $\|T\| \leq \lambda\|t\|$. Analogously, we will say that it uniformly $\mathcal{A}$-lifts if there exists $\lambda>0$ such that, for every Banach space $V$, every $t \in \mathcal{A}(V, Z)$ can be lifted to a $T \in \mathcal{A}(V, X)$ through $q$ with $\|T\| \leq \lambda\|t\|$.

Observe that when $\mathcal{A}$ is a closed ideal of $\mathfrak{L}$ then $\mathcal{A}$-splitting and uniform $\mathcal{A}$ splitting coincide; as well as $\mathcal{A}$-lifting and uniform $\mathcal{A}$-lifting. This obviously fails for non-closed ideals: for instance, when $\mathfrak{F}$ is the ideal of finite rank operators, all exact sequences $\mathfrak{F}$-split while, as it will be clear after definition 2 , not all exact sequences uniformly $\mathfrak{F}$-split.

In this paper we study when an exact sequence $\mathcal{A}$-splits or $\mathcal{A}$-lifts for the following choices of $\mathcal{A}$ : the ideal $\mathfrak{F}$ of finite rank operators, A of approximable operators, $\mathfrak{K}$ of compact operators, $\mathfrak{W}$ of weakly compact operators and $\mathfrak{L}$ of all linear continuous operators; for their associated polynomial ideals $\mathcal{P}_{\mathfrak{K}}$ of compact polynomials and $\mathcal{P}_{\mathfrak{W}}$ of weakly compact polynomials; and for their associated classes of holomorphic bounded mappings $\mathcal{H}_{\mathfrak{K}}$ and $\mathcal{H}_{\mathfrak{W}}$ (see sections 3 and 4 ).

The paper is organized as follows: Section 3 contains the main results about extension and lifting of operators. Given an exact sequence $0 \rightarrow Y \stackrel{i}{\rightarrow} X \stackrel{q}{\rightarrow} Z \rightarrow 0$ of Banach spaces, the Hahn-Banach theorem guarantees that the restriction operator $i^{*}: X^{*} \rightarrow Y^{*}\left(i^{*}\left(x^{*}\right)=\left.x^{*}\right|_{Y}\right)$ is surjective. We show that the existence of a linear continuous operator $s: Y^{*} \rightarrow X^{*}$ such that $i^{*} s=I d_{Y^{*}}$ is equivalent to any of the following conditions (see Theorem 3): the sequence locally splits (see Definition 2), uniformly $\mathfrak{F}$-splits, A-splits, $\mathfrak{K}$-splits, $\mathfrak{W}$-splits, uniformly $\mathfrak{F}$-litfs or A-lifts. If moreover $Z$ has the Bounded Approximation Property (in short, BAP), the previous conditions will be shown to be also equivalent to $\mathfrak{K}$-lifts. This result unifies and extends Kaballo [28, Thm. 3.4] and Fakhoury [20, Thm. 3.1]. It is then proved that $\mathfrak{K}$-splitting and $\mathfrak{K}$-lifting re not equivalent. The BAP has a decisive role in the lifting of operators. In Proposition 1 it is proved: A separable Banach space $Z$ has the $B A P$ if and only if for every exact sequence $0 \rightarrow Y \rightarrow X \rightarrow Z \rightarrow 0 \mathfrak{K}$-spliting and $\mathfrak{K}$-litfing are equivalent.

Equipped with these results and the representation of polynomials by symmetric tensor products, section 4 is devoted to prove extension/lifting results for polynomials and holomorphic bounded mappings. The injective tensor product $\check{\otimes}_{\varepsilon}$ (resp. projective $\hat{\otimes}_{\pi}$ ) has deep connections with vector functions spaces since it often occurs that injective (resp. projective) tensor product spaces can be represented as 
vector function spaces (see [4, 28, 16, 21]). We will prove that the notions locally

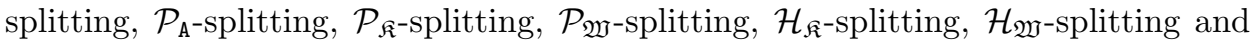
$\mathcal{P}_{\mathrm{A}}$-lifting are all equivalent. If moreover $Z$ has the BAP, the previous conditions are also equivalent to $\mathcal{P}_{\mathfrak{K}}$-lifting and $\mathcal{H}_{\mathfrak{K}}$-lifting (see Theorem 4 ). In Section 5 we provide characterizations of $\mathcal{L}_{1}$ and $\mathcal{L}_{\infty}$-spaces that include and extend those of Lindenstrauss-Rosenthal [32, Thm. 4.1] using compact operators and GonzálezGutiérrez [23, Thms. 2 and 4] using compact polynomials and Domanski [19, Thm. 4].

Section 6 contains the most interesting counterexamples in the paper. Its motivation is to make explicit the difference between "extending operators belonging to a certain operator ideal to operators of the same operator ideal" and the same property for liftings. This problem responds to a very natural homological problem as we explain in Section 7. The connection between the extension/lifting problem for operator ideals has appeared, often not explicitly, in many papers (see e.g., 32, 19]). We will call an operator ideal $\mathfrak{A}$ balanced when extension and lifting are equivalent for operators in $\mathfrak{A}$. For technical reasons explained in Section 6, this notion is only meaningful for injective and surjective (see below for the definition) operator ideals. We will then study the balanced character of the main classes of injective and surjective operator ideals appearing in the literature: compact, weakly compact, unconditionally convergent, separable and Rosenthal operators. The ideal of compact operator is the only one balanced.

Section 7 puts previous results in a homological perspective, which helps the interested reader to understand the motivations and nature of the results presented.

\section{Preliminaries}

For general information about operator ideals we suggest [34] or, more friendly, 17. Recall that an operator ideal $\mathcal{A}$ is a subclass of the class $\mathfrak{L}$ such that for all Banach spaces $V$ and $X$ its components $\mathcal{A}(V, X)=\mathfrak{L}(V, X) \cap \mathcal{A}$ satisfy: $\mathcal{A}(V, X)$ is a linear subspace of $\mathfrak{L}(V, X)$ which contains the finite rank operators and enjoys the ideal property: for $u \in \mathfrak{L}(V, X), t \in \mathcal{A}(X, W), w \in \mathfrak{L}(W, Y)$, the composition $w t u \in \mathcal{A}(V, Y)$. An operator ideal $\mathcal{A}$ is injective (resp. surjective) whenever given an operator $t \in \mathfrak{L}(V, Y)$ and an injection $i: Y \rightarrow X$ (resp. surjection $q: X \rightarrow Z$ ) then $t \in \mathcal{A}(V, Y)$ if and only if $i t \in \mathcal{A}(V, X)$ (resp. $t \in \mathcal{A}(Z, V)$ if and only if $t q \in \mathcal{A}(X, V)))$.

All necessary background information and unexplained notation about polynomials and holomorphic mappings can be found in [18; more specific information about homogeneous polynomial ideals can be seen in 35, 5. For information about homological algebra we address the reader to [26], while a sounder background on the theory of exact sequence of Banach spaces can be found in [13. Recall that a Banach space $X$ is said to have the Bounded Approximation Property (in short, BAP) if there is a constant $C>0$ such that for every $\epsilon>0$ and each compact subset $K$ of $X$, there is a finite rank operator $T$ in $X$ with $\|T\|<C$, such that $\|T x-x\| \leq \epsilon$, for each $x \in K$.

The push-out construction. Let us recall the push-out construction from the theory of exact sequences. Given two operators $i: Y \rightarrow X$ and $j: Y \rightarrow E$ their push-out is the space $P O=(E \times X) / \Delta$, where $\Delta=[\overline{(j y,-i y): y \in Y}]$ endowed 
with the quotient topology. Let $v: E \rightarrow P O$ and $w: X \rightarrow P O$ be the operators $v(m)=[(m, 0)]$ and $v(x)=[(0, x)]$. One has $v j=w i$. Moreover, given any Banach space $W$ and operators $a: E \rightarrow W$ and $b: X \rightarrow W$ so that $a j=b i$ there is a unique operator $u: P O \rightarrow W$ given by $u[m, x]=a m+b x$ such that $u v=a, u w=b$ and $\|u\| \leq \max \{\|a\|,\|b\|\}$. In particular, this means (see [13, 1.2,1.3]) that given an exact sequence $0 \rightarrow Y \stackrel{i}{\rightarrow} X \rightarrow Z \rightarrow 0$ and an operator $j: Y \rightarrow E$ the push-out of $i, j$ yields a commutative diagram

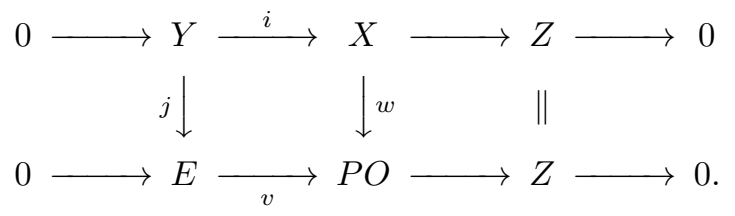

The following lemma (see [13, 1.3]) establishes the fundamental connection of the push-out construction regarding extension/lifting problems:

Lemma 1. In a push-out diagram,

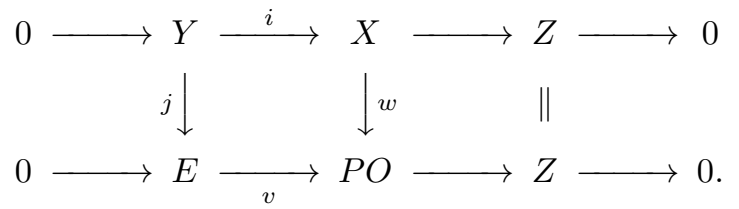

the lower sequence splits if and only if $j$ extends to $X$; that is, there is an operator $T: X \rightarrow E$ such that $T i=j$.

\section{Locally SPlitting Vs. $\mathcal{A}$-SPlitting}

Kalton introduced in 29 the notion of locally splitting for an exact sequence as follows (see also Fakhoury [20]):

Definition 2. An exact sequence $0 \rightarrow Y \stackrel{i}{\rightarrow} X \stackrel{q}{\rightarrow} Z \rightarrow 0$ is said to locally split if there exists a constant $\lambda>0$ such that for every finite dimensional subspace $E \subset X$ there exists a linear continuous operator $T_{E}: E \rightarrow Y$ such that $T_{E \mid E \cap Y}=i d_{E \cap Y}$ and $\left\|T_{E}\right\| \leq \lambda$. We will also say that $Y$ is locally complemented in $X$ when the corresponding exact sequence $0 \rightarrow Y \stackrel{i}{\rightarrow} X \stackrel{q}{\rightarrow} X / Y \rightarrow 0$ locally splits.

The Principle of Local Reflexivity of Lindenstrauss and Rosenthal 32, Thm. 3.1.], says that every Banach space is locally complemented in its bidual. Also, it is well known that every Banach space is locally complemented in its ultrapowers. Other results about local complementation are the following:

If $Y$ is an $\mathcal{L}_{\infty}$-space and $Y \subset X$ (or $X / Y$ is an $\mathcal{L}_{1}$-space) then $Y$ is locally complemented in $X$ (see [29]).

Thus, local splitting corresponds to uniform $\mathfrak{F}$-splitting. One moreover has:

Theorem 3. (Mainly Kalton-Fakhoury) Let $0 \rightarrow Y \rightarrow X \rightarrow Z \rightarrow 0$ be an exact sequence. The following are equivalent:

(1) The sequence locally splits.

(2) The sequence uniformly $\mathfrak{F}$-splits.

(3) The sequence uniformly $\mathfrak{F}$-litfs.

(4) The sequence $\mathfrak{K}$-splits. Equivalently, uniformly $\mathfrak{K}$-splits. 
(5) The sequence $\mathfrak{W}$-splits. Equivalently, uniformly $\mathfrak{W}$-splits

(6) The sequence A-splits. Equivalently, uniformly A-splits.

(7) The sequence A-lifts. Equivalently, uniformly A-lifts.

If, moreover, $Z$ has the BAP then assertions (1) to (7) are also equivalent to

(8) The sequence $\mathfrak{K}$-lifts. Equivalently, uniformly $\mathfrak{K}$-lifts.

Proof. The equivalences (1) to (4) were proved by Kalton [29] (and by Fakhoury [20, III] with other terminology). Kalton also shows [29, Thm. 3.5] that a sequence locally splits if and only if its dual sequence splits. From this (5) can be easily derived: on one hand, it is clear that $\mathfrak{W}$-splitting implies $\mathfrak{K}$-splitting; on the other hand, if the sequence $0 \rightarrow Y \stackrel{i}{\rightarrow} X \rightarrow Z \rightarrow 0$ locally splits, its dual sequence splits, hence also its bidual sequence $0 \rightarrow Y^{* *} \stackrel{i^{* *}}{\rightarrow} X^{* *} \rightarrow Z^{* *} \rightarrow 0$ splits. Let $p: X^{* *} \rightarrow Y^{* *}$ be a linear continuous projection. If $t: Y \rightarrow E$ is a weakly compact operator, its bidual $t^{* *}$ still is weakly compact and $E$-valued. Thus, $t^{* *} p: X^{* *} \rightarrow E$ is a weakly compact extension of $t$ and the sequence $\mathfrak{W}$-splits (see also [20,3.1]). The equivalence with (6) is also simple: since every approximable operator is compact, (4) implies (6). And since A is closed, A-splitting implies uniform A-splitting, hence uniform $\mathfrak{F}$-splitting, which is (2).

Observe now that $\mathfrak{K}$-lifting is not in the list, so the equivalence with (7) is new and has to be proved. That (7) implies (2) is clear, so we show that (2) implies (7). Assertion (7) exactly amounts showing that given any Banach space $V$ the induced sequence

$$
0 \longrightarrow \mathrm{A}(V, Y) \stackrel{i_{\circ}}{\longrightarrow} \mathrm{A}(V, X) \stackrel{q_{\circ}}{\longrightarrow} \mathrm{A}(V, Z) \longrightarrow 0,
$$

in which $i_{\circ}$ (resp. $q_{\circ}$ ) denote the operators "left-composition with $i$ " (resp. with $q$ ), is also exact. Thus, assume (2). That the sequence uniformly $\mathfrak{F}$-lifts implies that, for each Banach space $V$, the sequence of normed spaces

$$
0 \longrightarrow \mathfrak{F}(V, Y) \stackrel{i_{\circ}}{\longrightarrow} \mathfrak{F}(V, X) \stackrel{q_{\circ}}{\longrightarrow} \mathfrak{F}(V, Z) \longrightarrow 0
$$

is topologically exact (i.e.; $q_{\circ}$ is an open map). We show that the sequence formed by the completion of those spaces, namely,

$$
0 \longrightarrow \mathrm{A}(V, Y) \longrightarrow \mathrm{A}(V, X) \longrightarrow \mathrm{A}(V, Z) \longrightarrow 0
$$

is also an exact sequence. Let $j: \mathfrak{F}(V, Y) \rightarrow \mathrm{A}(V, Y)$ denote the canonical embedding. Making the push-out of the couple $j, i_{\circ}$ one gets the commutative diagram

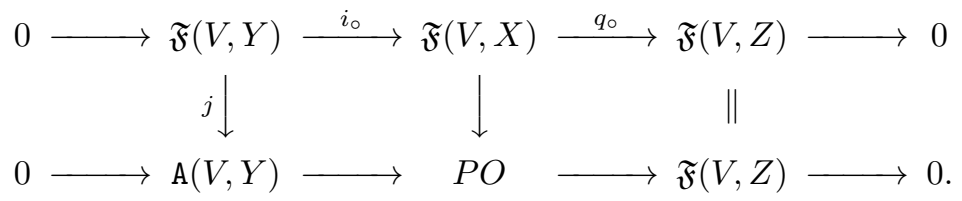

The universal property of the push-out applied to the operators $i_{\circ}: \mathrm{A}(V, Y) \rightarrow$ $\mathrm{A}(V, X)$ and $j: \mathfrak{F}(V, X) \rightarrow \mathrm{A}(V, X)$ (the canonical embedding) yields the existence of the operator $u: P O \rightarrow \mathrm{A}(V, X)$ given by $u[a, F]=i_{\circ} a+F$ and a commutative 
diagram

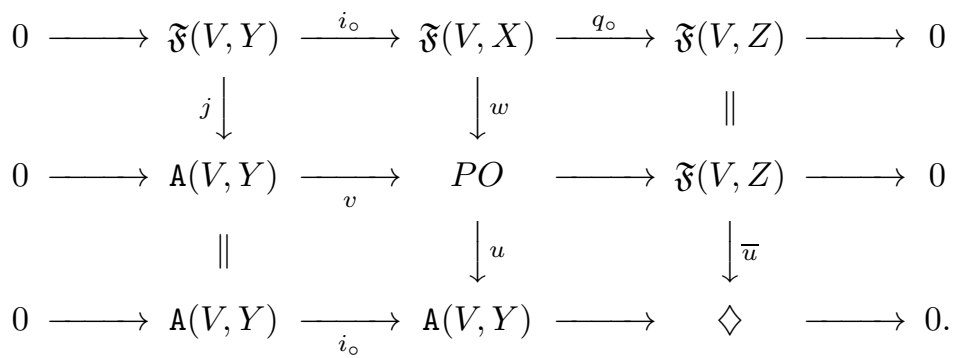

The operator $u$ has dense range since $u w$ is the canonical dense-range embedding $j: \mathfrak{F}(V, X) \rightarrow \mathrm{A}(V, X)$; so $\bar{u}$ must also have dense range. And since $\diamond$ is complete, it must be the completion $\mathrm{A}(V, Z)$ of $\mathfrak{F}(V, Z)$.

The equivalence with (8) follows from this since when $Z$ has the BAP then for every Banach space $V$ one has the identity $\mathfrak{K}(V, Z)=\mathrm{A}(V, Z)$.

The following proposition explains why $\mathfrak{K}$-lifting is not in the list of Theorem 3 .

Proposition 1. A separable Banach space $Z$ has the $B A P$ if and only if for every exact sequence $0 \rightarrow Y \rightarrow X \rightarrow Z \rightarrow 0 \mathfrak{K}$-spliting and $\mathfrak{K}$-litfing are equivalent.

Proof. Recall that James and Lindenstrauss [33, 1.d.3] proved that if $Z$ is a separable Banach space there exists a separable Banach space $X$ such that both $X$ and $X^{* *}$ have basis and moreover $X^{* *} / X=Z$. So there is an exact sequence

$$
0 \longrightarrow X \longrightarrow X^{* *} \longrightarrow Z \longrightarrow 0
$$

which locally splits by the Principle of Local Reflexivity [32, Thm. 3.1]. If $Z$ fails the BAP, there exists a compact non-approximable operator $K \in \mathfrak{K}(Z, Z)$ [16, 5.3]. If there would exist a compact lifting $\widetilde{K}: Z \rightarrow X^{* *}$ of $K$ then $\widetilde{K}$, hence also $K$, would be approximable.

It therefore follows that $\mathfrak{K}$-splitting and $\mathfrak{K}$-lifting are not equivalent notions. It is not hard to see that $\mathfrak{K}$-lifting and $\mathfrak{W}$-lifting are also non-equivalent, even in the presence of the BAP: indeed, it is shown in [30] (see also [9, Thm. 2.3.]) the existence of a nontrivial exact sequence

$$
0 \longrightarrow C[0,1] \longrightarrow X \longrightarrow \ell_{2} \longrightarrow
$$

or else, it is obtained in 27] the existence of nontrivial exact sequences

$$
0 \longrightarrow c_{0} \longrightarrow X \longrightarrow \ell_{2}(I) \longrightarrow 0 \text {. }
$$

In both cases, recall - see also 29, 32- that any exact sequence $0 \rightarrow Y \rightarrow X \rightarrow$ $Z \rightarrow 0$ in which $Y$ is an $\mathcal{L}_{\infty}$-space locally splits.

\section{EXTENSION AND LIFTING OF POLYNOMiALS AND HOLOMORPHiC MAPPings}

For the polynomial version of Theorem 3 the basic idea is to reduce the problem for polynomials and holomorphic maps to a problem on linear operators.

We denote by $\mathcal{P}\left({ }^{n} X\right)$ the Banach space of all continuous $n$-homogeneous polynomials on $X$, where the norm is given by $\|P\|=\sup \{P(x):\|x\| \leq 1\}$. The naturalisometric predual of $\mathcal{P}\left({ }^{n} X\right)$ is the projective symmetric tensor product $\hat{\otimes}_{\pi_{s}}^{n, s} X$ and 
the mapping $x \rightarrow x^{n}=\otimes^{n} x$ is a "universal" continuous $n$-homogeneous polynomial on $X$ : for every $P \in \mathcal{P}\left({ }^{n} X\right)$, there is a unique linearization $\widehat{P} \in \mathcal{L}\left(\hat{\otimes}_{\pi_{s}}^{n, s} X\right)$ with the same norm, such that $P(x)=\widehat{P}\left(x^{n}\right)$ for every $x \in X$ (see [21, 2.2]). Similarly, we have the vector-valued version of the previous identity $\mathcal{P}\left({ }^{n} X, V\right)=\mathcal{L}\left(\hat{\otimes}_{\pi_{s}}^{n, s} X, V\right)$.

Let $X$ be a complex Banach space. We denote by $\mathcal{H}_{b}(X)$ the Fréchet space of all holomorphic mappings on $X$ that are bounded on the bounded subsets of $X$. Let $\mathcal{H}_{\mathfrak{K}}(X)$ (resp. $\left.\mathcal{H}_{\mathfrak{W}}(X)\right)$ be the space of the holomorphic bounded functions in $X$ that are compact (respectively $w$-compact).

Let $f$ be a holomorphic function on $X$. The Taylor series

$$
f(x) \sim \sum_{n=1}^{\infty} \frac{d^{n} f(0)}{n !}(x) \quad\left(d^{n} f(0) \in \mathcal{P}\left({ }^{n} X\right) \simeq \mathcal{L}_{s}\left({ }^{n} X\right)\right) .
$$

decomposes $f$ as a formal sum $\sum_{n} P_{n}$ where $P_{n} \in \mathcal{P}\left({ }^{n} X\right)$. It is well known that $f \in \mathcal{H}_{\mathfrak{K}}(X)$ if and only if $P_{n} \in \mathcal{P}_{\mathfrak{K}}\left({ }^{n} X\right)$ for all $n$ (same for $\mathcal{H}_{\mathfrak{W}}(X)$ ), see [24, Prop. 5] or [3].

The definitions and general properties of projective and injective tensor product spaces can be found in [16, and 21] for the symmetric tensor product. In particular, the injective tensor product $\check{\otimes}_{\varepsilon}$ has deep connections with vector functions spaces since it often occurs that injective tensor product spaces can be represented as vector function spaces. Some examples for this assertion: $X \check{\otimes}_{\varepsilon} Y$ is always a subspace of $\mathfrak{K}_{w^{*}}\left(X^{*}, Y\right)$-the space of compact weak*-continuous operators- with equality when $X^{*}$ or $Y$ have BAP; also, the space $C(K, X)$ of continuous $X$-valued functions on a compact space $K$ coincides with $C(K) \check{\otimes}_{\varepsilon} X$; and the space $\mathcal{P}_{\mathrm{A}}\left({ }^{n} Y, X\right)$ coincides with $\left(\check{\otimes}_{\varepsilon, s}^{n} Y^{*}\right) \check{\otimes}_{\varepsilon} X([16,5.3])$. Further examples of distribution spaces in locally vector spaces can be found in [4, 28. Especially interesting for us is the identification

$$
\left(V \check{\otimes}_{\varepsilon} Z\right)^{*}=\mathcal{I}\left(V, Z^{*}\right)
$$

of the dual of the injective tensor product as the space $\mathcal{I}\left(V, Z^{*}\right)$ of integral operators, see $[16, \S 3 . \S 4$.] and the identification

$$
\left(V \hat{\otimes}_{\pi} Z\right)^{*}=\mathfrak{L}\left(V, Z^{*}\right)
$$

of the dual of the projective tensor product as the space $\mathfrak{L}\left(V, Z^{*}\right)$ of all operators.

It is well-known that the tensorization $V \check{\otimes}_{\varepsilon}-$ of an exact sequence is not necessarily exact (see in Section 7 the notions of left-exact and exact functor). Kaballo [28] defines an exact sequence $0 \rightarrow Y \rightarrow X \rightarrow Z \rightarrow 0$ of locally convex spaces to be an $(\varepsilon L)$-triple when, for every Banach space $V$, the tensorized sequence

$$
0 \longrightarrow V \check{\otimes}_{\varepsilon} Y \longrightarrow V \check{\otimes}_{\varepsilon} X \longrightarrow 0
$$

is exact. In the category of Banach spaces one has:

Proposition 2. Let $0 \rightarrow Y \rightarrow X \rightarrow Z \rightarrow 0$ be an exact sequence. The following are equivalent:

(1) The sequence is an $(\varepsilon L)$-triple.

(2) The sequence locally splits.

(3) For every Banach space $V$, the sequence

$$
0 \longrightarrow V \check{\otimes}_{\varepsilon} Y \longrightarrow V \check{\otimes}_{\varepsilon} X \longrightarrow 0
$$

is exact and locally splits. 
(4) For every Banach space $V$, the sequence

$$
0 \longrightarrow V \hat{\otimes}_{\pi} Y \longrightarrow V \hat{\otimes}_{\pi} X \longrightarrow V \hat{\otimes}_{\pi} Z \longrightarrow 0
$$

is exact and locally splits.

Proof. The equivalence between (1) and (2) was obtained by Kaballo [28, Thm. 2.2]. To see that (1) implies (3) it is enough to check that the adjoint operator $(I d \otimes q)^{*}$ : $\left(V \check{\otimes}_{\varepsilon} Z\right)^{*} \rightarrow\left(V \check{\otimes}_{\varepsilon} X\right)^{*}$ admits a linear continuous projection. Let $r$ be a projection for $q^{*}$; i.e., $r \circ q^{*}=I d_{Z^{*}}$. Under the identification of the dual of the injective tensor space with the space of integral operators the operator $(I d \otimes q)^{*}$ becomes left-composition with $q^{*}$. Thus, the operator $R: \mathcal{I}\left(V, X^{*}\right) \rightarrow \mathcal{I}\left(V, Z^{*}\right)$ given by $R(I)=r \circ I$ induces a projection through $(I d \otimes q)^{*}$. The case of the projective tensor product is analogous using the corresponding identification $\left(V \hat{\otimes}_{\pi} Z\right)^{*}=\mathcal{L}\left(V, Z^{*}\right)$. That (3),(4) imply (2) is clear since $V \check{\otimes}_{\varepsilon} Z=V \hat{\otimes}_{\pi} Z=Z$ when $V=\mathbb{K}$.

A few more facts about the so-called Aron-Berner extension for polynomials will be required. If $Y$ is a locally complemented subspace of $X$, it is then clear that there exists a linear continuous section $s: Y^{*} \rightarrow X^{*}$ extending operators (i.e.; $i^{*} s=I d_{Y^{*}},\left[29\right.$, Th. 3.5]). The operator $s$ induce -just using induction on $n^{-}$ continuous linear maps

$$
A B: \mathcal{P}\left({ }^{n} Y\right) \rightarrow \mathcal{P}\left({ }^{n} X\right) .
$$

This is the well-known Aron-Berner extension. We will use the notation $A B(P)=$ $\bar{P}$. Different descriptions of the Aron-Berner extension can be seen in 2, 11, 22, 40. The operator $A B$ is a section for the restriction operator $R: \mathcal{P}\left({ }^{n} X\right) \rightarrow \mathcal{P}\left({ }^{n} Y\right)$. Thus, the following exact sequence locally splits

$$
0 \longrightarrow \hat{\otimes}_{\pi, s}^{n} Y \stackrel{\otimes i}{\longrightarrow} \hat{\otimes}_{\pi, s}^{n} X \longrightarrow\left(\hat{\otimes}_{\pi, s}^{n} X\right) /\left(\hat{\otimes}_{\pi, s}^{n} Y\right) \longrightarrow 0 .
$$

About what types of polynomials are preserved by the Aron-Berner extension, it is clear that polynomials of finite type, approximable, compact and weakly compact polynomials are preserved (see [12, 40] for more classes of polynomials).

We now define the Aron-Berner extension for vector-valued polynomials. Let $Y, V$ be Banach spaces. If $\phi \in V^{*}$, the operator

$$
A B: \mathcal{P}\left({ }^{n} Y ; V\right) \rightarrow \mathcal{P}\left({ }^{n} X, V^{* *}\right)
$$

is defined by composition $A B(P)(x)(\phi)=(\overline{\phi \circ P})(x)$ (see [11, 12, 40]). In general, $A B$ does not take its values in $V$. It is clear that if $P \in \mathcal{P}\left({ }^{n} Y ; V\right)$ has weakly compact associated linear operator $T_{P}: Y \rightarrow \mathcal{P}\left({ }^{n-1} Y ; V\right)$ then $A B(P)$ is $V$-valued (see [12, Section 2.3]). Since the classes of finite type, approximable, compact and weakly compact polynomials all have weakly compact associated linear operator, their respective Aron-Berner extensions are $V$-valued (see 12 for details and the consideration of other classes of polynomials).

We are thus ready to obtain extension/lifting theorems for polynomials and holomorphic functions.

Theorem 4. Let $0 \rightarrow Y \stackrel{i}{\rightarrow} X \stackrel{q}{\rightarrow} Z \rightarrow 0$ be an exact sequence of Banach spaces. The following are equivalent:

(1) The sequence locally splits. 
(2) The sequence $\mathcal{P}_{A}$-splits.

(3) The sequence $\mathcal{P}_{A}$-lifts.

(4) The sequence $\mathcal{P}_{\mathfrak{K}}$-splits,

(5) The sequence $\mathcal{H}_{\mathfrak{K}}$-splits.

(6) The sequence $\mathcal{P}_{\mathfrak{W}}$-splits

(7) The sequence $\mathcal{H}_{\mathfrak{W}}$-splits.

If, moreover, $Z$ has the BAP, they are also equivalent to

(8) The sequence $\mathcal{P}_{\mathfrak{K}}$-lifts.

(9) The sequence $\mathcal{H}_{\mathfrak{K}}$-lifts.

Proof. $(1) \Rightarrow(3)$. To lift approximable polynomials it is enough to apply Proposition 2 and tensorize with $\mathcal{P}_{\mathrm{A}}(V)$ since $\mathcal{P}_{\mathrm{A}}\left({ }^{n} V, X\right)=\mathcal{P}_{\mathrm{A}}\left({ }^{n} V\right) \check{\otimes}_{\varepsilon} X([16,5.3])$.

$(1) \Rightarrow(2)$ Let $P \in \mathcal{P}_{f}\left({ }^{n} Y ; V\right)$ be a polynomials of finite type, say $P=\sum_{i=1}^{m} \phi_{i}^{n} y_{i}$ where $y_{i} \in Y$ and $\phi_{i} \in Y^{*}$. Then $A B(P)=\sum_{i=1}^{m} s(\phi)_{i}^{n} y_{i}$. It is follows that $A B: \mathcal{P}_{f}\left({ }^{n} Y ; V\right) \rightarrow \mathcal{P}_{f}\left({ }^{n} X ; V\right)$ is a complemented embedding. Using the arguments of the proof of Theorem 3 , the continuity of $A B$ and the completeness of $V$ yield (2).

$(1) \Rightarrow(4)$ and $(6)$. For the case of $\mathcal{P}_{\mathfrak{K}}$-splitting and $\mathcal{P}_{\mathfrak{W}}$-splitting, let $P \in$ $\mathcal{P}\left({ }^{n} X, V\right)$ and denote by $\widehat{P} \in \mathcal{L}\left(\hat{\otimes}_{\pi, s}^{n} X, V\right)$ the linearization of $P$ transforming compact polynomials (resp. $w$-compact) into compact operators (resp. $w$-compact) and viceversa (see [36, Lemma 4.1]). Since the sequence locally splits, the AronBerner extension provides the locally splitting of the sequence (see [11, 2.6])

$$
0 \longrightarrow \hat{\otimes}_{\pi, s}^{n} Y \stackrel{\otimes i}{\longrightarrow} \hat{\otimes}_{\pi, s}^{n} X \longrightarrow Q=\left(\hat{\otimes}_{\pi, s}^{n} X\right) /\left(\hat{\otimes}_{\pi, s}^{n} Y\right) \longrightarrow 0 .
$$

For a given polynomial $P \in \mathcal{P}_{\mathfrak{K}}\left({ }^{n} Y, V\right)$ (resp. $\mathcal{P}_{\mathfrak{W}}\left({ }^{n} Y, V\right)$ ), one gets that the linearization of $\widehat{P}$ can be extended as in the diagram

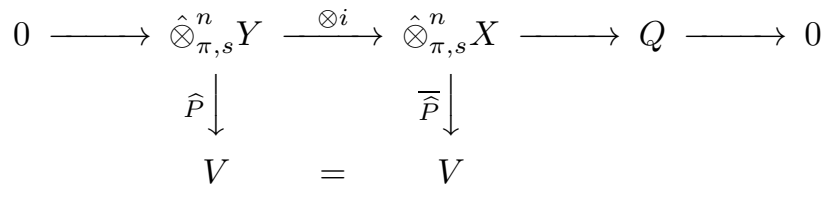

since the tensorized sequence $\mathfrak{K}$-splits (resp. $\mathfrak{W}$-splits) by Theorem 3 ,

$(1) \Rightarrow(5)$ and $(7)$. Let us show now the case of holomorphic functions. Let $f \in \mathcal{H}_{\mathfrak{K}}(Y, V)$ and let $f(x)=\sum_{k} \frac{d^{k} f(0)}{k !}(x)$ be its Taylor series, where $d^{k} f(0) \in$ $\mathcal{P}_{\mathfrak{K}}\left({ }^{n} X, V\right)$ (see [3, 18]). Then we may define the extension operator $\Phi: \mathcal{H}_{\mathfrak{K}}(Y, V) \rightarrow$ $\mathcal{H}_{\mathfrak{K}}(X, V)$ as

$$
\Phi(f)=\bar{f}=\sum_{k} \frac{\overline{d^{k} f}(0)}{k !} .
$$

The convergence follows from [12, Lemma 3.1] or [40] (see 2, 3, 18, for the scalar case). The same argument works for the case $\mathcal{H}_{\mathfrak{W}}(Y, V)$ (see [3, 12]). To obtain the converse, we just recall that the operators are exactly the polynomials of degree 1. We may also easily check that the map $s: Y^{*} \rightarrow X^{*}$ given by $s\left(y^{*}\right)=d\left(\Phi\left(y^{*}\right)\right)(0)$ is a section for $i^{*}: X^{*} \rightarrow Y^{*}$. 
$(1) \Rightarrow(8)$. If $Z$ has the BAP then one gets $\mathcal{P}_{\mathfrak{K}}\left({ }^{n} V, Z\right)=\mathcal{P}_{A}\left({ }^{n} V, Z\right)([16,5.3])$, and the result follows from (3).

Given a closed injective operator ideal $\mathcal{U} \subseteq \mathcal{W}$ the theorem above can be extended to the classes $\mathcal{P}_{\mathcal{U}}$ using the polynomial factorization given in [25, Cor. 5]. See the comment after [12, Cor. 2.7.] for details. Taking this into account, Theorem 4 yields.

Corollary 1. Let $0 \rightarrow Y \stackrel{i}{\rightarrow} X \stackrel{q}{\rightarrow} Z \rightarrow 0$ be an exact sequence of Banach spaces. The following are equivalent:

(1) The sequence locally splits

(2) For every dual Banach space $V^{*}$ with the $B A P$ the functor $\mathcal{P}_{\mathfrak{K} w^{*}}\left({ }^{n} V^{*},-\right)$ transforms it into an exact sequence.

(3) For every dual Banach space $V^{*}$ with the BAP the functor $\mathcal{H}_{\mathfrak{K} w^{*}}\left(V^{*},-\right)$ transforms it into an exact sequence.

Proof. It is well-known [16, 21] that if $V^{*}$ has the BAP then also $\left(\check{\otimes}_{s, \varepsilon}^{n} V\right)^{*}$ has the BAP. The result now follows from the identification

$$
\left(\check{\otimes}_{s, \varepsilon}^{n} V\right) \check{\otimes}_{\varepsilon}-=\mathcal{P}_{\mathfrak{K} w^{*}}\left({ }^{n} V^{*},-\right) .
$$

\section{Characterization of $\mathcal{L}_{\infty}$-SPaCes and $\mathcal{L}_{1}$-Spaces}

A Banach space $X$ is said to be an $\mathcal{L}_{\infty, \lambda}$-space (resp. $\mathcal{L}_{1, \lambda}$-space) if every finite dimensional subspace $E \subset X$ is contained in a finite dimensional subspace $F \subset X$ that is $\lambda$-isomorphic to $\ell_{\infty}^{\operatorname{dim} F}$ (resp. $\ell_{1}^{\operatorname{dim} F}$ ). If no reference to $\lambda$ is necessary we will simply say that $X$ is an $\mathcal{L}_{\infty}$ (resp. $\mathcal{L}_{1}$-space). Lindenstrauss and Rosenthal characterize in [32, Thm. 4.1.] the $\mathcal{L}_{\infty}$ (resp. $\left.\mathcal{L}_{1}\right)$-spaces through the $\mathfrak{K}$-splitting (resp. $\mathfrak{K}$-lifting) of exact sequences (equivalences $(1) \Leftrightarrow(2)$ in Theorems 5 and 6 below), while González and Gutiérrez in [23] extend the result using $\mathcal{P}_{\mathfrak{K}}$-splitting (resp. $\mathcal{P}_{\mathfrak{K}}$-lifting). We provide next a unified approach to these results and several generalizations.

Indeed, $\mathcal{L}_{\infty}$ and $\mathcal{L}_{1}$-spaces are necessarily involved in our study of extension/lifting of operators. To show why, recall that a Banach space $X$ is said to be injective if every exact sequence $0 \rightarrow X \rightarrow \diamond \rightarrow \boldsymbol{\phi} \rightarrow 0$ splits. It is on the other hand wellknown that a Banach space is an $\mathcal{L}_{\infty}$-space if and only if its bidual space is injective [32, p. 335]. One therefore has the following characterization of $\mathcal{L}_{\infty}$-spaces.

Theorem 5. Let $Y$ be a Banach space. The following conditions are equivalent:

(1) $Y$ is an $\mathcal{L}_{\infty}$-space.

(2) Every exact sequence $0 \rightarrow Y \rightarrow \diamond \rightarrow \boldsymbol{\Lambda} \rightarrow 0$ locally splits.

(3) Every exact sequence $0 \rightarrow Y \rightarrow \diamond \rightarrow \boldsymbol{M} \rightarrow 0 \mathcal{A}$-lifts for any of the choices $\mathcal{A}=\mathfrak{K}, \mathcal{P}_{\mathfrak{K}}$ or $\mathcal{H}_{\mathfrak{K}}$.

(4) Each of the functors $Y \check{\otimes}_{\varepsilon}-, \mathfrak{K}_{w^{*}}\left(Y^{*},-\right), \mathcal{P}_{\mathfrak{K} w^{*}}\left({ }^{n} Y^{*},-\right)$ or $\mathcal{H}_{\mathfrak{K} w^{*}}\left(Y^{*},-\right)$ transform exact sequences into exact sequences.

Proof. The equivalence between (1) and (2) is thus clear from the comments before the statement of the theorem and Kalton's characterization of locally splitting.

$(1) \Rightarrow(3)$ Let $K: Z \rightarrow \boldsymbol{\phi}$ be a compact operator. Take an index set $\Gamma$ for which there exists a linear continuous surjection $Q: \ell_{1}(\Gamma) \rightarrow Z$. Form then the 
commutative diagram

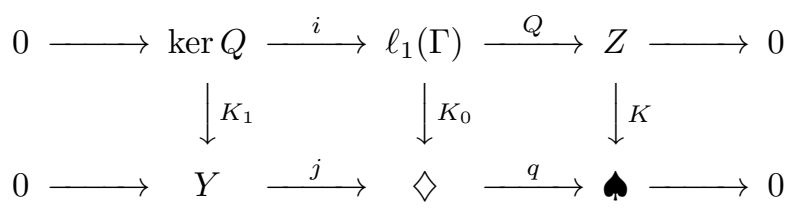

where we can assume that $K_{0}$ is a compact lifting of $K Q$ with $\left\|K_{0}\right\| \leq(1+\varepsilon)\|K\|$, see [32, Thm. 4.1.]. Thus, $K_{1}$ must also be compact. Consider then a compact extension $\widetilde{K}_{1}: \ell_{1}(\Gamma) \rightarrow Y$ of $K_{1}$, which yields a compact operator $j \widetilde{K}_{1}-K_{0}$ : $\ell_{1}(\Gamma) \rightarrow \diamond$. Since $\left(j \widetilde{K}_{1}-K_{0}\right) i=0$, the operator $j \widetilde{K}_{1}-K_{0}$ must factorize through a (necessarily compact) operator $\widetilde{K}: Z \rightarrow \diamond$ which is the desired lifting of $K$ we were looking for.

The exactness of $Y \check{\otimes}_{\varepsilon}-$ can be found in [28, Thm. 1.5]; see also [16, p. 307]). For the remaining assertions one just need to recall ([37, p. 68] and [21, 3.1]) that when $Y$ is an $\mathcal{L}_{\infty}$ space then also $\check{\otimes}_{\varepsilon, s}^{n} Y$ is an $\mathcal{L}_{\infty}$-space and that the equality $\mathcal{P}_{\mathfrak{K} w^{*}}\left({ }^{n} Y^{*},-\right)=\left(\check{\otimes}_{s, \varepsilon}^{n} Y\right) \check{\otimes}_{\varepsilon}-$ holds since $Y$ is complemented in $\check{\otimes}_{\varepsilon, s}^{n} Y$.

For the holomorphic case, we consider that the decomposition of $f$ as a formal sum $\sum_{n} P_{n}$ where $P_{n} \in \mathcal{P}_{\mathfrak{K} w^{*}}\left({ }^{n} Y^{*},-\right)$ ( [24, Prop. 5].) The result follows from the proof of Theorem 4 .

$(3) \Rightarrow(1)$ Given a compact operator $K: X \rightarrow Y$, consider a commutative diagram

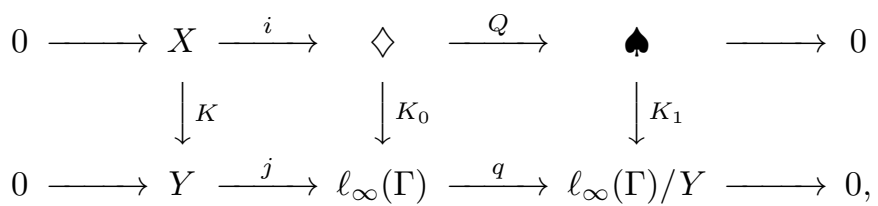

where $K_{0}, K_{1}$ can be chosen to be compact. A similar argument as above works.

(4) This follows from the Proposition 2 and Corollary 1.

\section{Remark 1.}

a) The case (3) for $\mathfrak{K}$ (resp. (2) for $\mathfrak{W})$ appear in Domanski [19, Thm. 4], equivalence between (b.i) and (b.ii) (resp. between (a.i) and (a.ii)).

b) González and Gutiérrez show in [23, Remark 1] that $\mathcal{P}_{\mathfrak{K}}\left({ }^{n}-, Y\right)$ does not necessarily transforms exact sequences into exact sequences, even when $Y$ is an $\mathcal{L}_{\infty}$-space.

Dually, it is well-known that a Banach space is is an $\mathcal{L}_{1}$-space if and only if its dual space is injective (32]). One therefore has.

Theorem 6. Let $Z$ be a Banach space. The following conditions are equivalent

(1) $Z$ is an $\mathcal{L}_{1}$-space.

(2) Every exact sequence $0 \rightarrow \mathbf{\phi} \rightarrow \diamond \rightarrow Z \rightarrow 0$ locally splits.

(3) Every exact sequence $0 \rightarrow \mathbf{\Lambda} \rightarrow \diamond \rightarrow Z \rightarrow 0 \mathcal{A}$-lifts for any of the choices $\mathcal{A}=\mathfrak{K}, \mathcal{P}_{\mathfrak{K}}$ or $\mathcal{H}_{\mathfrak{K}}$. 
(4) Every exact sequence $0 \rightarrow R \rightarrow \diamond \rightarrow Z \rightarrow 0$ in which $R$ is reflexive splits.

(5) Each of the functors $Z \hat{\otimes}_{\pi}-, \mathfrak{K}(Z,-), \mathcal{P}_{\mathfrak{K}}\left({ }^{n} Z,-\right)$ or $\mathcal{H}_{\mathfrak{K}}(Z,-)$ transform exact sequences into exact sequences.

Proof. The equivalence between (1) and (2) is clear, and since $\mathcal{L}_{1}$ spaces have the BAP, the equivalence with (3) is also clear by Theorem 4. (4) is consequence of the $\mathfrak{W}$-splitting and the Davis-Figiel-Johnson-Pełczyński factorization of weakly compact operators [16, 9.6]. The equivalence (1) and (5) follows from [16, 3.9]. The proof for $\mathcal{P}_{\mathfrak{K}}\left({ }^{n} Z,-\right)$ follows from the equality $\mathcal{P}_{\mathfrak{K}}\left({ }^{n} Z,-\right)=\left(\left(\check{\otimes}_{\varepsilon, s}^{n} Z^{*}\right) \check{\otimes}_{\varepsilon}-\right)$ and from the fact that $\left(\check{\otimes}_{\varepsilon, s}^{n} Z^{*}\right)$ is an $\mathcal{L}_{\infty}$-space $([37$, p. 68] and [21, 3.1]).

Observe that (4) has no analogue for $\mathcal{L}_{\infty}$-spaces since $\mathcal{L}_{\infty}$-spaces are not characterized by $\mathfrak{W}$-lifting, as previous examples show. $\mathcal{L}_{1}$-spaces are not characterized by $\mathfrak{W}$-lifting either: given a sequence $0 \rightarrow \operatorname{ker} q \rightarrow \ell_{1} \stackrel{q}{\rightarrow} L_{1}[0,1] \rightarrow 0$, no embedding $\ell_{2} \rightarrow L_{1}[0,1]$ can be lifted through $q$ since every operator $\ell_{2} \rightarrow \ell_{1}$ must be compact.

\section{EXTENSION VS. LIFTING FOR OPERATOR IDEALS}

The difference between $\mathfrak{K}$-splitting and $\mathfrak{K}$-lifting was remarked by Theorem 3 , In the proof of Theorem 5 commutative diagrams such as

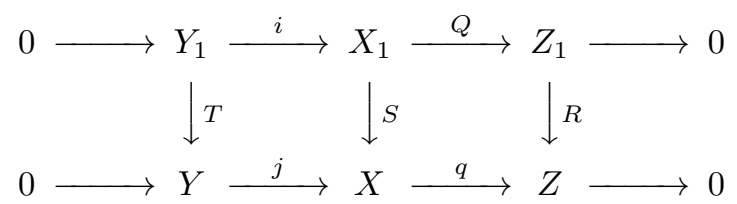

appeared. It is well known that for such diagrams [13, 1.2, 1.3] -see also [19, Prop. 1]- the operator $R$ can be lifted to an operator $Z_{1} \rightarrow X$ if and only if $T$ can be extended to an operator $X_{1} \rightarrow Y$. In the proof of Theorem 5 there was moreover exhibited a certain symmetry between " $R$ admits a compact lifting" and "T admits a compact extension". One could ask about the exact nature of such extension/lifting behaviour and if the same happens for other operator ideals.

Definition 7. An operator ideal $\mathfrak{A}$ will be called balanced when given a commutative diagram

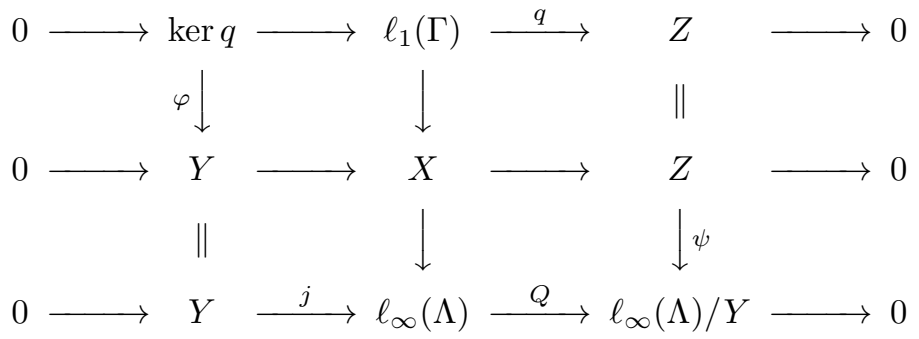

the operator $\varphi$ can be chosen in $\mathfrak{A}$ if and only if $\psi$ can be chosen in $\mathfrak{A}$.

Observe that this does not mean that $\psi$ must be in $\mathfrak{A}$ whenever $\varphi$ is in $\mathfrak{A}$; it rather means that whenever $\varphi$ is in $\mathfrak{A}$ the operator $j \varphi$ admits an extension $\ell_{1}(\Gamma) \rightarrow \ell_{\infty}(\Lambda)$ whose induced operator $Z \rightarrow \ell_{\infty}(\Lambda) / Y$ is in $\mathfrak{A}$ and viceversa: whenever $\psi$ is in $\mathfrak{A}$ the operator $\psi q$ admits a lifting $\ell_{1}(\Gamma) \rightarrow \ell_{\infty}(\Lambda)$ whose restriction $\operatorname{ker} q \rightarrow Y$ is in $\mathfrak{A}$.

For reasons hidden in the homological roots of the problem (see Section 7) this notion is interesting only when the ideal $\mathfrak{A}$ is injective and surjective (see [34]; see 
also Section 7). Classical injective and surjective operator ideals appearing in the literature (see [34]) are the ideals $\mathfrak{F} ; \mathfrak{K} ; \mathfrak{W} ; \mathfrak{U}$ (unconditional summing operators); $\mathfrak{R}$ (Rosenthal operators) and $\mathfrak{X}$ (separable range operators). We determine now their balanced character.

Proposition 3. The ideal $\mathfrak{K}$ is balanced. The ideals $\mathfrak{W}, \mathfrak{U}, \mathfrak{X}$ and $\mathfrak{R}$ are not balanced.

Proof. The ideal $\mathfrak{K}$ of compact operators is balanced. Assume that $\psi$ is compact, which makes $\psi q$ also compact. An observe that an operator $T: \ell_{1}(\Gamma) \rightarrow X$ is compact if and only if for every countable part $\mathbb{N} \subset \Gamma$ the restriction $T_{\mid \ell_{1}(\mathbb{N})}$ is compact. Which occurs if and only if $\left\{T\left(e_{n}\right)\right\}_{n}$ is a relatively compact set. In conclusion, that an operator $T i: \ell_{1}(\Gamma) \rightarrow X$ is compact if and only if given any sequence of $e_{i}^{\prime} s$ there is a subsequence $\left(e_{j}\right)$ for which $\left(T e_{j}\right)$ converges. Let $\omega$ be a continuous (non-linear) selection for the quotient map $\ell_{\infty}(\Lambda) \rightarrow \ell_{\infty}(\Lambda) / Y$, which exists by the Bartle-Graves selection theorem [13, p. 52]. The operator $\Phi: \ell_{1}(\Gamma) \rightarrow \ell_{\infty}(\Lambda)$ defined by $\Phi\left(e_{i}\right)=\omega \varphi q e_{i}$ is therefore compact, and its restriction to ker $q$ yields a compact operator $\varphi: \operatorname{ker} q \rightarrow Y$ that makes commutative the diagram above. Conversely, assume that $\varphi$ is compact. Lindenstrauss's characterizations of $\mathcal{L}_{\infty}$ spaces [31] yield a compact extension $K: \ell_{1}(\Gamma) \rightarrow \ell_{\infty}(\Lambda)$ of $j \varphi$. The operator $Q K$ factorizes as $Q K=\psi q$ with $\psi$ compact.

The ideal $\mathfrak{W}$ of weakly compact operators is not balanced. Using a result of Bourgain and Pisier [7, Thm. 2.1], every separable Banach space $X$ can be embedded into a separable $\mathcal{L}_{\infty}$-space $\mathcal{L}_{\infty}(X)$ in such a way that the corresponding quotient $\mathcal{L}_{\infty}(X) / X$ has the Schur property (namely, weakly convergent sequences are norm convergent). Consider then an exact sequence $0 \rightarrow D \rightarrow \ell_{1} \rightarrow \ell_{2} \rightarrow 0$, apply the Bourgain-Pisier construction to $D$ and combine both results in a push-out diagram

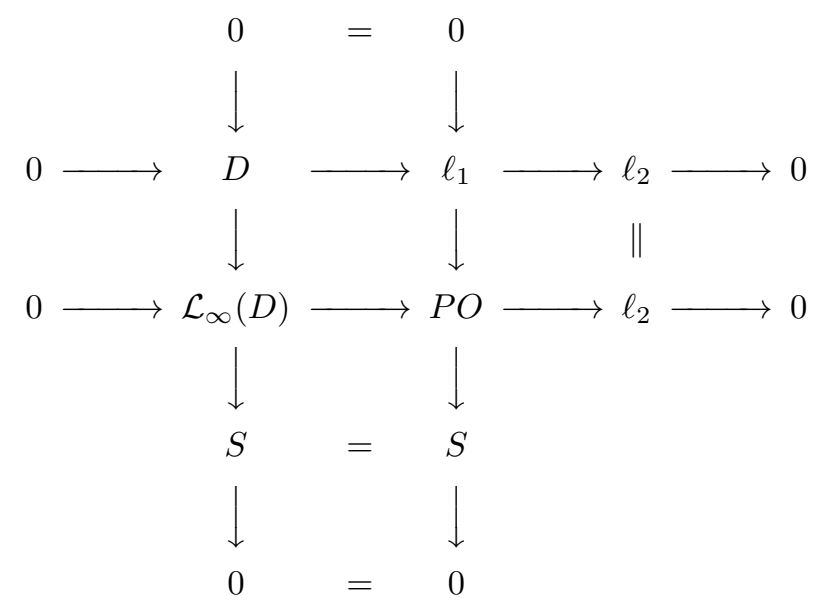

By a standard 3-space argument (see [13, Thm. 6.1.a]) the space $P O$ has the Schur property, hence the sequence $0 \rightarrow \mathcal{L}_{\infty}(D) \rightarrow P O \rightarrow \ell_{2} \rightarrow 0$ cannot split. Consider 
a commutative diagram

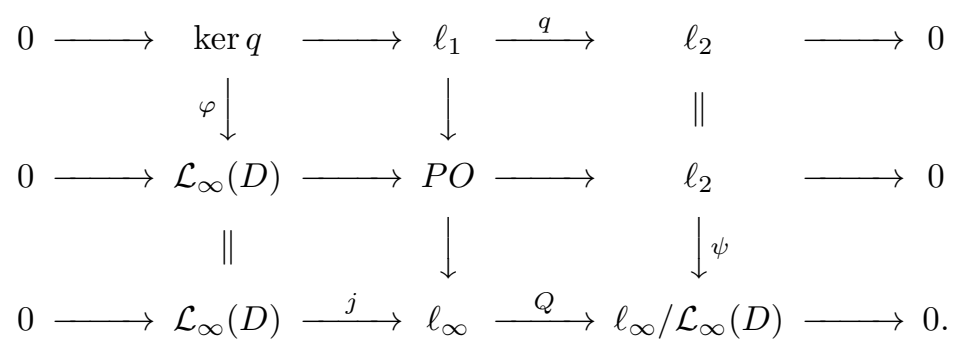

The operator $\psi$ is always weakly compact. However, the operator $\varphi$ cannot be weakly compact: since the space $\mathcal{L}_{\infty}(D)$ has the Schur property, every weakly compact operator with range $\mathcal{L}_{\infty}(D)$ must be compact, and could therefore be extended to any separable superspace. This would make the middle sequence split.

The ideal $\mathfrak{U}$ of unconditionally summing operators is not balanced. Recall that an operator $t: X \rightarrow Y$ belongs to $\mathfrak{U}(X, Y)$ (i.e., it is unconditionally summing) if and only if it is never an isomorphism on a copy of $c_{0}$. Recall from [30], or else [9], examples of nontrivial sequences

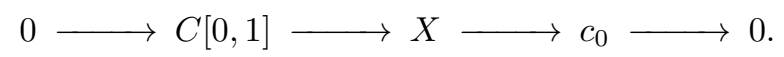

Consider then a commutative diagram

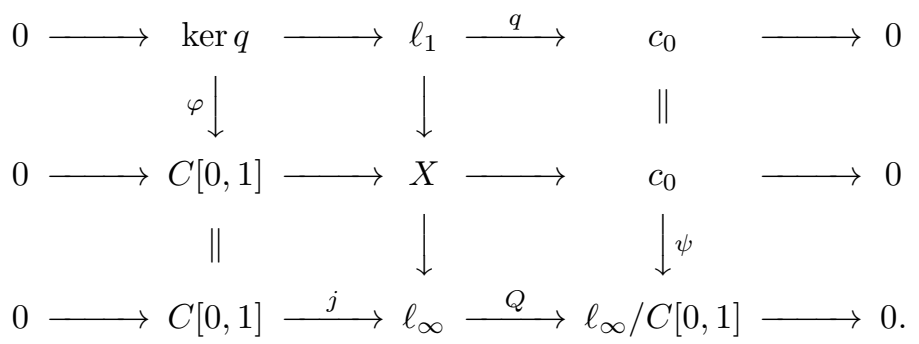

The operator $\varphi$ is always unconditionally summing since $\mathfrak{L}\left(\ell_{1}, Y\right)=\mathfrak{U}\left(\ell_{1}, Y\right)$ for every Banach space $Y$. On the other hand, every operator on $c_{0}$ is either weakly compact or an isomorphism on a copy of $c_{0}$ (see 33 ); i.e., $\mathfrak{U}\left(c_{0}, Y\right)=\mathfrak{W}\left(c_{0}, Y\right)$ for every Banach space $Y$; but weakly compact operators on $c_{0}$ must be compact, hence $\mathfrak{U}\left(c_{0}, Y\right)=\mathfrak{K}\left(c_{0}, Y\right)$ for every Banach space $Y$. This means that every unconditionally summing $\psi$ must be compact, hence, by Theorem 5, it can be lifted to an operator $c_{0} \rightarrow \ell_{\infty}$, which means that the middle sequence splits.

The ideal $\mathfrak{X}$ of separable range operators is not balanced. It is well-known the existence of nontrivial exact sequences (see [27])

$$
0 \longrightarrow c_{0} \longrightarrow X \longrightarrow \ell_{2}(\Gamma) \longrightarrow 0
$$


Consider a commutative diagram

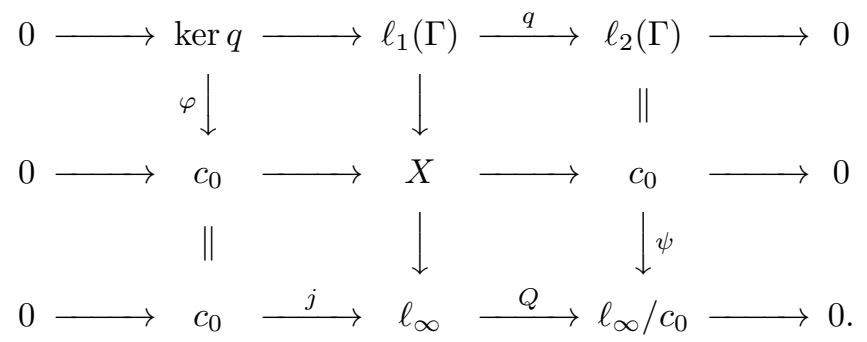

Every $\varphi$ with range $c_{0}$ has separable range. On the other hand, if some $\psi$ has separable range $S$ one gets a commutative diagram

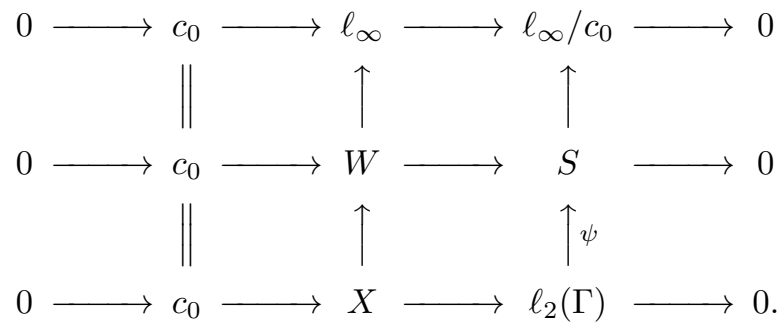

But Sobczyk's theorem [10] yields that the middle sequence splits; hence the lower sequence must also split.

The ideal $\mathfrak{R}$ of Rosenthal operators is not balanced. Recall that an operator $t$ is said to be a Rosenthal operator if it maps bounded sequences into sequences admitting weakly Cauchy subsequences. Consider an exact sequence $0 \rightarrow \operatorname{ker} q \rightarrow$ $\ell_{1} \stackrel{q}{\rightarrow} c_{0} \rightarrow 0$ and apply the Bourgain-Pisier construction [7, Thm. 2.1] to ker $q$ to get an exact sequence $0 \rightarrow \operatorname{ker} q \rightarrow \mathcal{L}_{\infty}(\operatorname{ker} q) \rightarrow S \rightarrow 0$ in which $S$ has the Schur property. Combine all this in a commutative push-out diagram

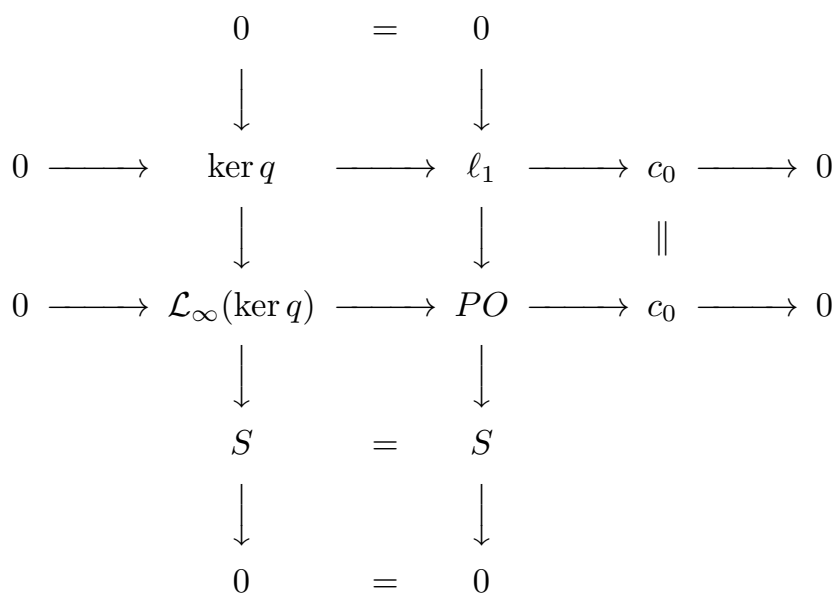

By a standard 3-space argument (see [13, Thm. 6.1.a]) the space $P O$ has the Schur property, hence the sequence $0 \rightarrow \mathcal{L}_{\infty}(\operatorname{ker} q) \rightarrow P O \rightarrow c_{0} \rightarrow 0$ cannot split. 
Consider now a commutative diagram

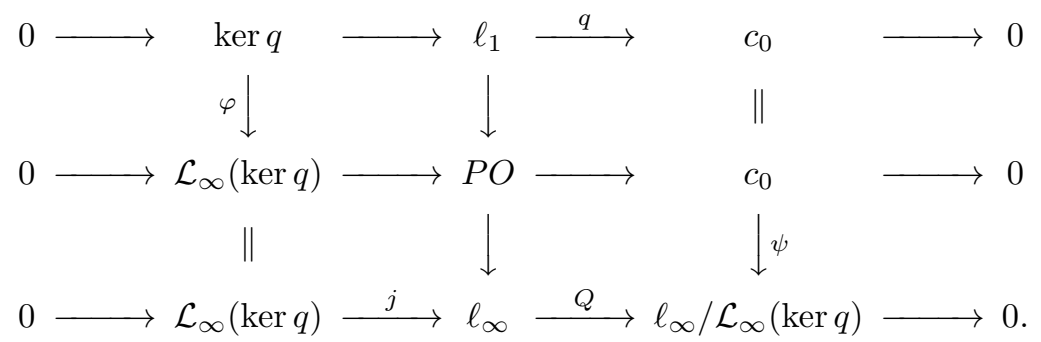

On one hand, $\mathfrak{L}\left(c_{0}, Y\right)=\mathfrak{R}\left(c_{0}, Y\right)$ for every Banach space $Y$. Hence $\psi$ must be a Rosenthal operator. On the other hand, assume that some $\varphi$ is a Rosenthal operator. Since weakly Cauchy sequences in Schur spaces are weakly convergent, and weakly convergent sequences are convergent, the operator $\varphi$ must be compact. Since the ideal $\mathfrak{K}$ is balanced, there must be some compact $\psi$ in the diagram above. But since $\ell_{\infty} / \mathcal{L}_{\infty}(K)$ is an $\mathcal{L}_{\infty}$-space [32, Prop. 5.2.(c)], it has the BAP, and thus Proposition 11 yields that $\psi$ can be lifted to an operator $\mathfrak{L}\left(c_{0}, \ell_{\infty}\right)$, which in turn means that the middle sequence must split.

\section{Appendix. The homological language}

Several of our results admit a homological formulation, which can be clearer for those familiar with the language and methods of homological algebra. We make now a brief exposition.

7.1. Exact functors. Let $\mathcal{A}$ be a suitable space of functions/operators. Given a fixed Banach space $X$ the functor $\mathcal{A}(X,-)$ assigns to a Banach space $Y$ the space $\mathcal{A}(X, Y)$ and to each linear continuous operator $\tau: Y \rightarrow Y_{1}$ the "composition-by -the-left" map, namely $\tau_{\circ}: \mathcal{A}(X, Y) \longrightarrow \mathcal{A}\left(X, Y_{1}\right)$ defined by $\tau_{\circ}(f)=\tau \circ f$. The fact that the direction of arrows is preserved is usually remarked by saying that the functor is covariant. Dually, for fixed $Y$ one has the contravariant functor $\mathcal{A}(-, Y)$ assigning to each Banach space $X$ the space $\mathcal{A}(X, Y)$ and to each linear continuous operator $\tau: X \rightarrow X_{1}$ the "composition-by -the-right" map, namely $\tau^{\circ}: \mathcal{A}\left(X_{1}, Y\right) \longrightarrow \mathcal{A}(X, Y)$ defined by $\tau^{\circ}(f)=f \circ \tau$.

A functor $\mathrm{F}$ is said to be left-exact (resp. right-exact) when given an exact sequence $0 \rightarrow Y \rightarrow X \rightarrow Z \rightarrow 0$ the induced sequence $0 \rightarrow \mathrm{F}(Y) \rightarrow \mathrm{F}(X) \rightarrow \mathrm{F}(Z)$ (resp. $\mathrm{F}(Y) \rightarrow \mathrm{F}(X) \rightarrow \mathrm{F}(Z) \rightarrow 0$ ) is exact. For instance, it is well known (see [16, 28]) that the functor $V \check{\otimes}_{\varepsilon}-$ (resp. $V \hat{\otimes}_{\pi}-$ ) is left-exact (resp. right-exact); or that given an injective and surjective operator ideal (see 34] $\mathcal{A}$ the functor $\mathcal{A}(X,-)$ is left-exact and the functor $\mathcal{A}(-, Y)$ is right-exact.

Definition 8. We say that a functor $F$ is semi-exact if it transforms locally splitting sequences into exact sequences. We will say it is locally exact if it transforms locally splitting sequences into locally splitting sequences.

Theorem 3 and Proposition 2 immediately yield.

Proposition 4. Given a Banach space $V$ one has:

- The functors $\mathfrak{K}(-, V)$ and $\mathfrak{W}(-, V)$ are semi-exact.

- The functors $A(V,-)$ and $A(-, V)$ are locally-exact. 
We have shown (combine Proposition 2, Theorem 4 and Aron-Berner extension) that

Corollary 2. The functors $\left(V \check{\otimes}_{\varepsilon}-\right)$ and $\left(V \hat{\otimes}_{\pi}-\right)$ are semi-exact if and only if they are locally exact.

7.2. Derivation. In many concrete situations, the involved functors $\mathcal{A}(Z,-)$ (resp. $\mathcal{A}(-, Y))$ are not exact. Homology theory was developed to study this case. The measure of how far a functor $\mathrm{F}$ is from being exact is given by the so-called derived functor of $\mathrm{F}$. The derivation process can be performed in different forms: using projective presentations (when they exist), injective presentations (when they exist), pull-back and push-out constructions, ... 26, 13.

Such is the case when one considers the category of Banach spaces and the functor(s) induced by the operator ideal $\mathfrak{L}$, namely $\mathfrak{L}(X,-)$ and $\mathfrak{L}(-, X)$. In which case the derived functor(s) are called Ext. See [8] for a detailed exposition. All this means that if one has a Banach space $Z$ and fixes a "projective presentation" of $Z$; namely, an exact sequence $0 \rightarrow \operatorname{ker} q \rightarrow \ell_{1}(I) \stackrel{q}{\rightarrow} Z \rightarrow 0$, and then fixes another Banach space $Y$ then there exists an exact sequence

$$
0 \longrightarrow \mathfrak{L}(Z, Y) \longrightarrow \mathfrak{L}\left(\ell_{1}(I), Y\right) \longrightarrow \mathfrak{L}(\operatorname{ker} q, Y) \longrightarrow \operatorname{Ext}_{\mathfrak{L}}^{p}(Z, Y) \longrightarrow 0 .
$$

This can be taken as the definition of the space $\operatorname{Ext}_{\mathfrak{L}}^{p}(Z, Y)$, which can be called the first derived functor of $\mathfrak{L}(-,-)$; or, to be more precise, the first projectively derived functor of $\mathfrak{L}(-,-)$. If, instead, one fixes an "injective presentation" of $Y$; namely, exact sequences $0 \rightarrow Y \rightarrow \ell_{\infty}(\Lambda) \rightarrow Q \rightarrow 0$, then there also exists an exact sequence

$$
0 \longrightarrow \mathfrak{L}(Z, Y) \longrightarrow \mathfrak{L}\left(Z, \ell_{\infty}(\Lambda)\right) \longrightarrow \mathfrak{L}(Z, Q) \longrightarrow \operatorname{Ext}_{\mathfrak{L}}^{i}(Z, Y) \longrightarrow 0 .
$$

Here $\operatorname{Ext}_{\mathfrak{L}}^{i}(-,-)$ can be called the first derived (injectively derived, to be precise) functor of $\mathfrak{L}(-,-)$. Classical homology theory starts with the fact [26] that injective and projective derivations coincide; i.e.:

$$
\operatorname{Ext}_{\mathfrak{L}}^{p}(Z, Y)=\operatorname{Ext}_{\mathfrak{L}}^{i}(Z, Y) .
$$

In fact, a non-written rule [26 asserts that when one is working in a reasonable category in which injective and projective presentations exist then the derived functors obtained via injective presentations and via projective presentations coincide.

What happens when the functor to be derived is not the one induced by the ideal $\mathfrak{L}$, but is induced by other operator ideals $\mathcal{A}$. Let us show that making relative homology with respect to an operator ideal requires to distinguish between injective and projective derivation. Indeed, one needs to ask the operator ideal to be injective (see 34]) in order to compute the relative Ext via injective presentations; and it has to be projective (see [34]) in order to compute the relative Ext using projective presentations. Let us define these functors to then show that they can be quite different.

Proposition 5. A projective operator ideal $\mathfrak{A}$ is left-exact.

This yields that given a projective operator ideal $\mathfrak{A}$, a Banach space $Z$ and an exact sequence $0 \rightarrow \operatorname{ker} q \rightarrow \ell_{1}(\Gamma) \stackrel{q}{\rightarrow} Z \rightarrow 0$, and given another Banach space $Y$ then there exists an exact sequence

$$
0 \longrightarrow \mathfrak{A}(Z, Y) \longrightarrow \mathfrak{A}\left(\ell_{1}(\Gamma), Y\right) \longrightarrow \mathfrak{A}(\operatorname{ker} q, Y) \longrightarrow \diamond \longrightarrow 0 .
$$


Straightforward computations show that $\diamond$ does not depend on the projective presentation of $Z$, and this allows us to define

$$
\operatorname{Ext}_{\mathfrak{A}}^{p}(Z, Y)=\diamond \text {. }
$$

Proposition 6. An injective operator ideal $\mathfrak{A}$ is right-exact.

This yields that given an injective operator ideal $\mathfrak{A}$, a Banach space $Y$ and an exact sequence $0 \rightarrow Y \rightarrow \ell_{\infty}(\Lambda) \rightarrow Q \rightarrow 0$, and given another Banach space $Z$ then there exists an exact sequence

$$
0 \longrightarrow \mathfrak{A}(Z, Q) \longrightarrow \mathfrak{A}\left(Z, \ell_{\infty}(\Lambda)\right) \longrightarrow \mathfrak{A}(Z, Y) \longrightarrow \diamond \longrightarrow 0 .
$$

Straightforward computations show that $\diamond$ does not depend on the injective presentation of $Y$, which allows us to define

$$
\operatorname{Ext}_{\mathfrak{A}}^{i}(Z, Y)=\diamond .
$$

We have thus arrived to the core of the reason behind the definition of balanced ideal. One has.

Proposition 7. An injective and surjective operator ideal $\mathfrak{A}$ is balanced if projective and injective derivation coincide; equivalently, if the functors $\operatorname{Ext}_{\mathfrak{A}}^{p}(\cdot, \cdot)$ and $\operatorname{Ext}_{\mathfrak{A}}^{i}(\cdot, \cdot)$ are naturally equivalent.

It is easy now to translate the results of the previous section to this language. For instance, the fact that $\mathfrak{K}$ is balanced means that $\operatorname{Ext}_{\mathfrak{K}}$ is well defined, while the fact that $\mathfrak{W}$ is not balanced means that one has to consider two functors Ext $t_{\mathfrak{W}}^{i}$ and $\operatorname{Ext}_{\mathfrak{W}}^{p}$, which can be very different. For instance, the examples in the previous section mean:

(1) $\operatorname{Ext}_{\mathfrak{W}}^{i}\left(\ell_{2}, \mathcal{L}_{\infty}(D)\right) \neq 0=\operatorname{Ext}_{\mathfrak{W}}^{p}\left(\ell_{2}, \mathcal{L}_{\infty}(D)\right)$.

(2) $\operatorname{Ext}_{\mathfrak{U}}^{i}\left(c_{0}, C[0,1]\right)=0 \neq \operatorname{Ext}_{\mathfrak{U}}^{p}\left(c_{0}, C[0,1]\right)$.

(3) $\operatorname{Ext}_{\mathfrak{X}}^{i}\left(\ell_{2}(\Gamma), c_{0}\right)=0 \neq \operatorname{Ext}_{\mathfrak{U}}^{p}\left(\ell_{2}(\Gamma), c_{0}\right)$.

(4) $\operatorname{Ext}_{\mathfrak{R}}^{i}\left(c_{0}, \mathcal{L}_{\infty}(\operatorname{ker} q)\right) \neq 0=\operatorname{Ext}_{\mathfrak{R}}^{p}\left(c_{0}, \mathcal{L}_{\infty}(\operatorname{ker} q)\right)$.

\section{REFERENCES}

[1] R. Aron, Extensions and lifting theorem for analytic mappings, In: Functional Analysis: survey and recent results. II. K. D. Bierstedt and B. Fuchssteiner, eds., North Holland ath. Studies. 38 (1980), 257-267.

[2] R. Aron and P. Berner, A Hahn-Banach extensions theorem for analytic mappings, Bull. Soc. Math. France 106 (1978), 3-24.

[3] R. Aron and M. Schottenloher, Compact holomorphic mappings on Banach spaces and the approximation property, J. Funct. Anal. 21 (1976), 7-30.

[4] K.D. Bierstedt, The $\varepsilon$-(tensor) product of (DFS)-space with arbitrary Banach spaces, pp. 3551 Functional Analysis (Proceedings of the Trier Work-shop, 1994), de Gruyter, Berlin, 1996, 35-51.

[5] G. Botelho, D. Pellegrino, and P. Rueda, On Composition Ideals of Multilinear Mappings and Homogeneous Polynomials, Publ. RIMS, Kyoto Univ. 43 (2007), 1139-1155.

[6] J. Bourgain and F. Delbaen, A class of special $\mathcal{L}_{\infty}$-spaces, Acta Math. 145 (1980), 155-176.

[7] J. Bourgain and G. Pisier, A construction of $\mathcal{L}_{\infty}$-spaces and related Banach spaces, Bol. Soc. Brasil. Mat. 14 (1983), 109-123.

[8] F. Cabello Sánchez and J.M.F. Castillo, The long homology sequence for quasi-Banach spaces, with applications, Positivity 8 (2004), 379-394.

[9] F. Cabello Sánchez, J.M.F. Castillo, N. J. Kalton, and D.T. Yost, Twisted sums with $C(K)$-spaces, Trans. Amer. Math. Soc. 355 (2003), 4523-4541. 
[10] F. Cabello Sánchez, J.M.F. Castillo, and D. Yost, Sobczyk's theorems from A to B, Extracta Math. 15 (2000) 391-420.

[11] F. Cabello Sánchez, R. García, and I. Villanueva, Extensions of multilinear operator on Banach spaces, Extracta Math. 15 (2000), 291-334.

[12] D. Carando and S. Lassalle, $E^{\prime}$ and it's relation with vector-valued functions on $E$, Ark. Mat. 42 (2004), 283-300.

[13] J.M.F. Castillo and M. Gonzáles, Three-space problems in Banach spaces, Lecture Notes in Mathematics 1667, Springer-Verlag (1997).

[14] J.M.F. Castillo, A. Defant, R. García, D. Pérez-García, J. Suárez, Local complementation and the extension of bilinear mappings, Math. Proc. Cambridge Philos. Soc. (to appear).

[15] R. Cilia and J. M. Gutiérrez, Extension and lifting of weakly continuous polynomials, Studia Math. 169 (3)(2005), 229-241.

[16] A. Defant A. and K. Floret, Tensor Norms and Operator Ideals, North-Holland Math. Studies 176.

[17] J. Diestel, H. Jarchow and A. Tonge, Absolutely summing operators, Cambridge Studies in Advanced Mathematics, 43, Cambridge University Press, 1995.

[18] S. Dineen, Complex Analysis on Infinite Dimensional Spaces, Monographs in Math., Springer, Berlin 1999.

[19] P. Domanski, Ideals of extendable and liftable operators, RACSAM 97 (2003), 229-241 .

[20] H. Fakhoury, Selections lineaires associees au Theoreme de Hahn-Banach, J. Fund. Anal. 11 (1972), 436-452.

[21] K. Floret, Natural norms on symmetric tensor products of normed spaces, Note Mat. 17 (1997), 153-188

[22] R. García, J. A. Jaramillo, and J. G. Llavona, The Aron-Berner extension, Goldstine's theorem and P-continuity, Math. Nachr. 284 (2011), 694-702.

[23] M. González and J. M. Gutiérrez, Extension and lifting of polynomials, Arch. Math. 81 (2003), 431-438.

[24] M. González and J. M. Gutiérrez, Surjective factorization of holomorphic mappings, Comment. Math. Univ. Carolin. 41 (2000) 469-476.

[25] M. González and J. M. Gutiérrez, Injective factorization of holomorphic mappings, Proc. Amer. Math. Soc. 127 (1999), no. 6, 1715-1721.

[26] E. Hilton and K. Stammbach, A course in homological algebra, Graduate Texts in Mathematics 4, Springer-Verlag 1970.

[27] W.B. Johnson and J. Lindenstrauss. Some remarks on weakly compactly generated Banach spaces. Israel J. Math. 17 (1974), 219-230.

[28] W. Kaballo, Lifting theorems for vector valued functions and the $\epsilon$-tensor products, K. D. Bierstedt, B. Fuchssteiner (eds.) Functional Analysis: Survey and Recent Resuts, North-Holland Publ. Comany (1977), 149-166.

[29] N.J. Kalton, Locally complemented subspaces and $\mathcal{L}_{p}$-spaces for $0<p<1$, Math. Nachr. 115 (1984), 71-97.

[30] N.J. Kalton, On subspaces of $c_{0}$ and extension of operators into $C(K)$-spaces, Q. J. Math. Oxford 52 (2001), 313-328.

[31] J. Lindenstrauss, On the extension of compact operators, Mem. Amer. Math. Soc. AMS 48 (1964).

[32] J. Lindenstrauss and H.P. Rosenthal, The $\mathcal{L}_{p}$-spaces, Israel J. Math. 7 (1969), 325-349.

[33] J. Lindenstraus and L. Tzafriri, Classical Banach spaces I, Springer-Verlag, Berlin, 92, 1977.

[34] A. Pietsch, Operator Ideal, Dtsch. Veralg d. Wissenschaften Berlin 1978, and North Holland Math. Library, 1980.

[35] A. Pietsch, Ideals of multilinear functionals, Proceedings of the Second International Conference on Operator Algebras, Ideals and Their Applications in Theoretical Physics, 185?199, Teubner-Texte, Leipzig, 1983.

[36] R. A. Ryan, Applications of topological tensor products to infnite dimensional holomorphy, Ph. D. Thesis, Trinity College, Dublin 1980.

[37] R. Ryan, Introduction to Tensor Products of Banach Spaces, Springer, London , 2002.

[38] A. Sobczyk, On the extension of linear transformations, Transactions of the American Mathematical Society 55 (1944) 153-169. 
[39] E.W. Straeuli, On Hahn-Banach extension for certain operator ideals, Arch. Math. (Basel) 47, no. 1 (1986), 49-54.

[40] I. Zalduendo, A canonical extension for analytic functións on Banch spaces, Trans. Amer. Math. Soc. 320 (1990), 747-763.

Departamento de Matemáticas, Facultad de Ciencias, Univ. de Extremadura, Avda ELVAS S/N 06011, BADAJOZ, EsPaña

E-mail address: castillo@unex.es

Departamento de Matemáticas, Facultad de Ciencias, Univ. de Extremadura, Avda ELVAS S/N 06011, BADAJOZ, EsPaÑA

E-mail address: rgarcia@unex.es

Departamento de Matemáticas, Escuela Politécnica de Cáceres, Univ. de Extremadura, Avda Universidad S/N 07001, CÁCERes, España

E-mail address: jesus@unex.es 